Cláudio R. Olinto

crolinto@yahoo.com.br

Member, $A B C M$

\section{Maria Luíza S. Indrusiak}

sperbindrusiak@via-rs.net

\section{Sérgio V. Möller}

Senior Member, ABCM

svmoller@ufrgs.br

Programa de PG em Engenharia Mecânica

Universidade Federal do Rio Grande do Sul 90050-170 Porto Alegre, RS, Brasil

\title{
Experimental Study of the Bistable Flow in Tube Arrays
}

Flow through circular cylinder arrays are commonly found in several engineering application as offshore structures, heat exchangers, transmission lines and chimneys, therefore the understanding of the several phenomena that occur due the interaction between flowing fluid and these structures is very important. This work analyzes experimentally the presence of phenomenon called bistable or biased flow, which is able to cause alternations on the flow modes in cylinder arrays. For the experimental work, two cylinders are placed side-by-side, or forming a tube bank with in line arrangement. The first arrangement corresponds to the case where the phenomenon is easily detected, and in line tube bank, the phenomenon has been also found. The experiments were performed in a wind channel using hot wire anemometry additionally to the classical statistic and spectral tools, wavelet transforms are used. The bistable flow can be an important generator of dynamic instabilities, since it alternates the lift and drag coefficients, thus alternating the structure dynamic response.

Keywords: Biased flow, tube bank, turbulent flow, hot wires

\section{Introduction}

Tube banks are usually found in the nuclear and process industries, being the most common geometry of heat exchangers. In experimental studies, tube banks are usually represented by arrays of cylinders. Several engineering applications, e.g. offshore structures, heat exchangers, transmission lines and chimneys, also use circular cylinders placed as close arrays.

Studies about the flow dynamic through circular cylinders placed side by side have shown a flopping phenomenon that produces random changes in the flow mode. Similar phenomenon has also been found in some tube banks. Thereby, new studies should be made to improve the understanding about flow induced vibration and fluid-structure interaction for the configurations where this phenomenon is present.

According to Zdravkovich e Stonebanks (1988) the leading feature of flow-induced vibration in tube banks is the randomness of dynamic responses of tubes. In spite of the fact that the tubes are all of equal size, have the same dynamic characteristics, are arranged in regular equidistant rows and are subjected to an uniform and steady flow, the dynamic response is non-uniform and random to such an extent, that the vibration of tubes resembles the Brownian motion of molecules.

Behind a tube row several wake sizes can be present and, consequently, several Strouhal numbers are expected. Therefore, the designers should be aware that a range of Strouhal numbers is relevant for any tube and not only two or a set of values as commonly used. Attention should be given also for the fact that a change between two different modes of flow can be an additional excitation mechanism on the tubes.

\section{Nomenclature}

$$
\begin{aligned}
& D=\text { cilinder diameter, } \mathrm{mm} \\
& f=\text { frequency, } \mathrm{Hz} \\
& f s=\text { sampling frequency, } \mathrm{kHz} \\
& p=\text { pressure, } \mathrm{Pa} \\
& R e=\text { Reynolds number } \\
& S t=\text { Strouhal number }(\mathrm{St}=\mathrm{fD} / \mathrm{V}) \\
& P=\text { Transversal pitch, } \mathrm{mm} \\
& P / D=\text { pitch to diameter ratio }
\end{aligned}
$$

Presented at ETT $2004-4^{\text {th }}$ Spring School on Transition and Turbulence September $27^{\text {th }}$ - October $1^{\text {st }}, 2004$, Porto Alegre, RS, Brazil.

Paper accepted: May, 2005. Technical Editor: Aristeu da Silveira Neto.

$$
\begin{aligned}
& V=\text { Velocity }, \mathrm{m} / \mathrm{s} \\
& x=\text { longitudinal distance }, \mathrm{mm}
\end{aligned}
$$

\section{Literature Review}

Sumner et al. (1999) studied the flow fields for two and three cylinders placed side-by-side for P/D-ratios between 1 and 6 and for Reynolds number varying between 500 and 3000 . They did not find the bistable phenomenon presence and the flow remained deflected consistently towards the same cylinder. The flow organization pattern was synchronized in anti-phase vortex formation. Inside the biased flow range two frequency peaks were detected. They attributed this behavior without bistable phenomenon to the experimental apparatus characteristics.

According to their study, the cross steady flow through equal diameter (D) circular cylinders placed side-by-side presents a wake with different modes depending on the distance between the centers of the cylinders $(\mathrm{P})$. For cylinders in contact $(\mathrm{P} / \mathrm{D}=1)$ a bluff body behavior is found and the shedding frequency is lower than that of a single cylinder, because of the increase of the distance between the free shear layers from both sides of the cylinders.

At small pitch ratios $(1,0<\mathrm{P} / \mathrm{D}<1.2)$ the bluff body behavior still occurs, but the higher-momentum fluid, which enters through the gap between cylinders, increases the base pressure and reduces the drag of both cylinders. The vortex-shedding frequency, however, tends to remain close to that observed for $P / D=1$. Furthermore, a single vortex street is still observed in the combined wake of the two cylinders, and shedding occurs only from the outside shear layers.

At large pitch ratios (P/D > 2.2) the biased flow is not present and the flow through the side-by-side circular cylinders behaves as the flow through isolated bluff bodies. However, some interaction or synchronization occurs between the two cylinders, predominantly as anti-phase vortex formation.

At intermediate pitch ratios $(1.2<\mathrm{P} / \mathrm{D}<2.0)$ the flow is characterized as a narrow near-wake behind a cylinder and a wide near-wake behind the other, which generate two dominant vortexshedding frequencies - the higher associated with the narrow wake and the lower with the wide wake. The gap flow deviates towards the cylinder with the narrow wake. In some cases, the biased gap flow is bistable and switches from one side to the other at irregular intervals of time. According previous studies, this pattern is independent from Reynolds number, and is not caused by cylinder misalignment or another external influence, but is an actual feature of the flow. Peschard \& Le Gal (1996) suggested the bistable behavior could be caused by turbulent perturbations from flow entrance. 
According to Kim and Durbim (1988) the transition between two asymmetric states is completely random and it is not associated with a natural frequency. The mean time interval between the transitions is on the order of $10^{3}$ times longer than the vortex shedding period. Studying the dimensionless mean time intervals between the switches, they observed that the time decreases as the Reynolds number increases. This is in accordance with Williamson (1985), who found a steady mean flow at $\operatorname{Re}=200$. As the Strouhal numbers are relatively independent from the Reynolds number, they concluded that there is no correlation between the vortex shedding and the bistable process.

Guillaume and LaRue (1999) classified the bistable regime according to its behavior:

- Quasi-stable behavior, the switches in the flow mode do not vary over the time. A large amplitude perturbation can cause the changing on average values, but the new values remain until another large perturbation is applied.

- Spontaneous flopping, the average values are always observed to alternate over the time between relatively high and low values, even when no large perturbation is applied to the flow field.

- Forced flopping, after the initial large perturbation is applied and flopping occurs, there is no observable difference between forced and spontaneous flopping.

The authors found also that the wake fluctuation pattern depends on the tubes supports, tunnel geometry and downstream interferences (probe supports).

Zdravkovich (1977) studied the two side-by-side tubes geometry and found that, associated to the wakes, there are two different drag coefficients and the base pressure switches between two extreme values. According to his observations, there also exists a resultant force acting perpendicular to the streamwise direction, which he called a lift force. The drag force acting on the cylinders is also different for each one, because of the different base pressures. It was verified that de sum of high and low drag forces in bistable regime is always less than twice the drag of a single cylinder.

In addition, Williamson (1985) concluded that, when the wake inclines towards to higher frequency side, the cylinder of this side experiments a larger drag force. He also included that the near-wake structure is confused for this asymmetric flow regime. His visualizations show that the inner vortices shedding from the gap are squeezed, distorted and amalgamated with the dominant outer vortices. Thus, at a certain distance from the cylinders, only the vortices generated at the outer surfaces of the pairs of cylinders are visualized.

Zdravkovich e Stonebanks (1988) studied the wake behind a tube row subjected to a perpendicular flow and concluded that it is formed by coalescent jet cells that suddenly change their pattern. This state, which he defined as metastable, can be due to the rearrangement of the cell pattern behind the row and is strongly dependent upon the number of tubes in the row.

For arrangements of two rows, the authors concluded that the biased gap flow disappears in the first row and is reestablished after the second row. They argued that it should be a typical feature behind any last row.

Le Gal et al. (1996) also studied the flow through a tube row. When the distance between the cylinder axes is larger than two cylinder diameters, they found an identical and anti-phase vortex shedding. Conversely, when this distance is less than two cylinder diameters, the jets between the tubes are deviated and the wake merges to form clusters. The cell sizes depend on the initial conditions. If the flow velocity is increased quickly from rest, several different patterns can be formed. Regions with wake oscillations can also appear.

According to Guillaume et al. (1999), who studied the flow through four and three in line tubes, in the intermediate wake three mean vortex-shedding frequencies may occur, the lowest associated to the wide wake, a second one with higher value, corresponding to the narrow wake and the highest, due the interaction between the lower and the second (intermediate) frequency. They also found that the wake fluctuation pattern depends on the tube supports, tunnel geometry and the downstream interference, presenting two peaks in the velocity spectra. Near the tubes $(\mathrm{x} / \mathrm{D}=2$, where $\mathrm{x}$ is the distance between the tube center line and the probe position) the peaks corresponds to $\mathrm{St}=0.354$ e 0.344 . These peaks are related to the narrow wake. For larger distances, the peaks are St $=0.1099$ and 0.1143 and are related to the wide wake.

Zhou et al. (2002) studied velocity and temperature fields in the turbulent wake of two tubes placed side by side. They experimented arrays with $\mathrm{P} / \mathrm{D}=1.5$ and 3.0 and compared the results with that of single tube wake. They calculated the vortex convection velocity through the maximum phase-average vorticity. In the spectral analysis they found a single frequency peak at $\mathrm{St}=0.11$ for $\mathrm{P} / \mathrm{D}=$ 1.5. For $\mathrm{P} / \mathrm{D}=3.0$ and single tube, they found a single frequency peak at $\mathrm{St}=0.21$, instead of two frequencies, as found by previous authors. According to their results, the vortex generation in the gap region is essential to form two vortex streets. A narrow gap can inhibit the internal vortex generation and thus two cylinders behave as a single structure generating just one vortex street.

Alam et al. (2003) investigated experimentally the flow through two cylinders placed side-by-side for $\operatorname{Re}=5.5 \times 10^{4}$, where the acting forces are insensible to the Reynolds number. They found, for intermediate P/D-ratios (1.2 to 2.2), the biased bistable regime and studied the fluctuating and steady forces characteristics. For P/D varying in the range 1 to 2.5 , two values for drag coefficients are found. They concluded that the decreasing of the wake width makes the drag coefficient to increase, and this is associated with the increasing of the gap velocity.

In the range $\mathrm{P} / \mathrm{D}=1.1$ to 2.5 , they found two lift coefficients always generating forces which tend to separate the cylinders (repulsion). Thus, they concluded that the narrow wake increases the drag force and decreases the lift force, whilst the wide wake decreases the drag force and increases lift. The lift force is in repulsion form for $\mathrm{P} / \mathrm{D}>1.2$. For $\mathrm{P} / \mathrm{D}=1.1$, the cylinder with narrow wake presents an attraction lift coefficient.

Alam et al. also demonstrate, using wavelet transform, the velocity energy spectra for two tubes with $\mathrm{P} / \mathrm{D}=1.7$, and concluded that, when a mode switch occur, an intermediate frequency may be present.

Indrusiak et al. (2003) studied transient flows through tube banks, using wavelet transforms, and found behind the third row a behavior with random change between the mean velocity values for both transient and steady flows. This intermittence is similar to bistable mode found for tubes placed side-by-side.

\section{Objectives}

The purpose of this paper is to describe the biased and bistable flow mode for two tubes placed side-by-side perpendicularly to an impinging flow and to show the relation between these phenomena and the intermittence found inside the tube bank.

\section{Experimental Technique}

The test section, shown schematically in Fig.1 is a rectangular channel, with $146 \mathrm{~mm}$ height and a width of $195 \mathrm{~mm}$. Air was the working fluid, driven by a centrifugal blower through a set of honeycombs and screens, before reaching the tube row with about 1 $\%$ turbulence intensity.

Before the tube row, a Pitot tube was placed at a fixed position, to measure the reference velocity for the experiments. The Reynolds 
number, calculated with the tube diameter $(32.1 \mathrm{~mm})$ and the entrance (reference) velocity is $\operatorname{Re}=3 \times 10^{4}$, for two tubes and $\mathrm{Re}=$ $1.5 \times 10^{4}$, for the tube bank.

Velocity and velocity fluctuations were measured by means of a DANTEC StreamLine constant temperature hot-wire anemometer. Pressure was measured by an ENDEVCO piezo-resistive pressure transducer, mounted inside one of the tubes of the row. Figure $2 \mathrm{a}$ shows the probe position for the experiments with two tubes side by side and Fig. 2b shows the location of the measurement probes in the tube bank, where 1 and 2 are the hot wire probes measuring respectively velocities named V1 and V2 and 3 is the pressure transducer.

Data acquisitions of pressure and velocity fluctuations were performed simultaneously by a Keithley DAS-58 A/D-converter board controlled by a personal computer.

The tube bank was mounted with 25 tubes having a $32.1 \mathrm{~mm}$ diameter placed vertically and distributed at 5 rows with 5 tubes each one. The pitch to diameter ratio it was $\mathrm{P} / \mathrm{D}=1.26$. The tube length was $146 \mathrm{~mm}$ and they were in close contact with both upper and lower horizontal walls. The tubes were rigid and not heated.

\section{Mathematical Tools}

To characterize the flows studied, the probability density function and four moments for the acquired time series are calculated, respectively: mean value, standard deviation, skewness and kurtosis. Auto and cross correlation functions, along with spectral analysis were also applied. The wavelet transform do not require the ergodic hypothesis and is used for the study of the transient flows. Wavelets can localise bistable phenomena in time and frequency domain. The continuous and discrete wavelet transforms are described in Indrusiak and Möller, 2004. The mathematical analysis was made with Matlab 5.3 software.

\section{Results}

\section{Two Tubes Placed Side-by-Side}

The biased flow effects and the bistable phenomenon were studied from time series obtained in the wind channel. Table 1 presents three acquisitions at distinct distances from the tubes centreline using two probes positioned as shown at Fig.2, for simultaneous acquisitions. The reference velocity during the experiments was $13.8 \mathrm{~m} / \mathrm{s}$, corresponding to a value of $3 \times 10^{4}$ for the Reynolds number.

The hot wire velocity signals, obtained immediately after the tubes, Fig. 3 (signal 1), show the presence of the bistable phenomenon and the two flow modes just by visual inspection. It can be viewed that about 15 mode switches occur during the interval of 130 seconds of the signal.

Table 1. Characteristics of the acquired signals.

\begin{tabular}{|c|c|c|c|c|}
\hline signal & $\mathrm{x}(\mathrm{mm})$ & $\mathrm{x} / \mathrm{D}$ & $\mathrm{fs}(\mathrm{kHz})$ & $\mathrm{t}(\mathrm{s})$ \\
\hline 1 & 20 & 0.62 & 1 & 131 \\
\hline 2 & 30 & 0.93 & 8 & 8.2 \\
\hline 3 & 70 & 2.18 & 1 & 65.5 \\
\hline
\end{tabular}

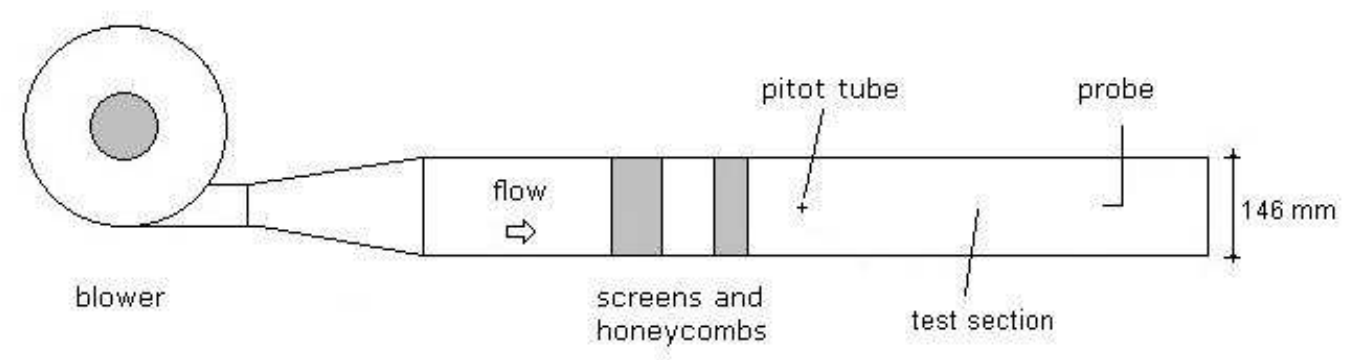

Figure 1. Schematic view of the wind tunnel.

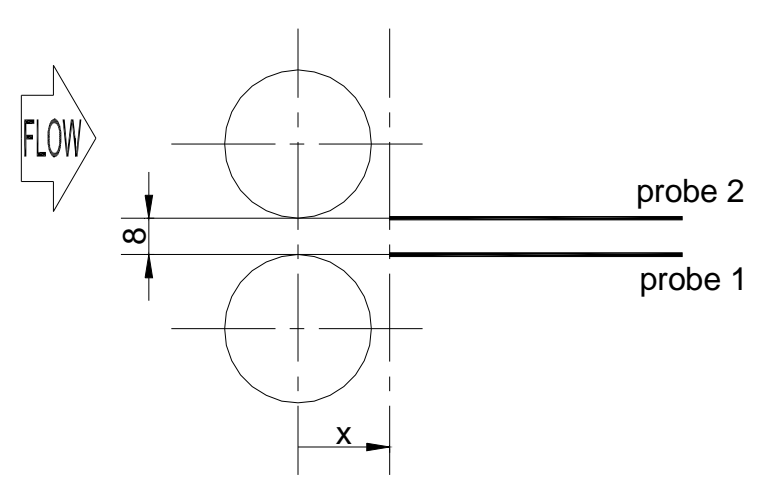

(a)

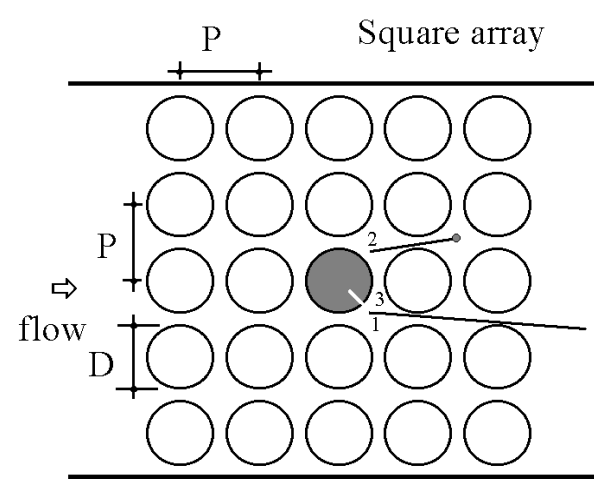

(b)

Figure 2. Tube arrays and measurement probes location. 


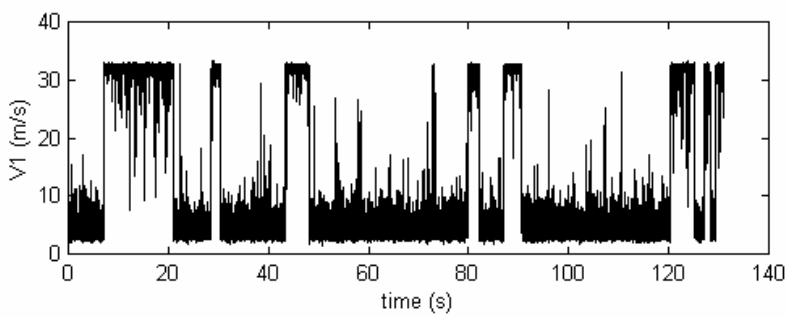

(a)

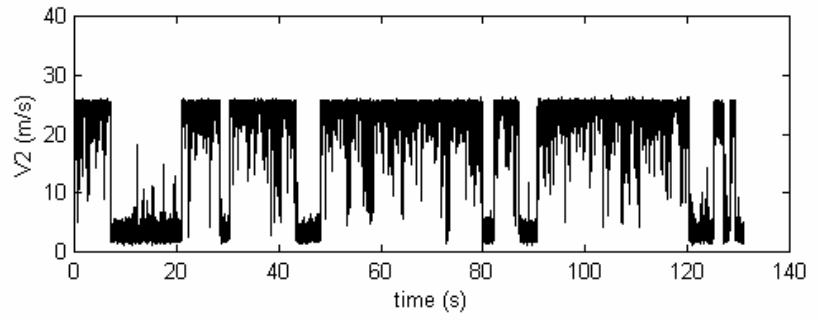

(b)

Figure 3. Instantaneous Velocities - signal 1; (a) V1; (b) V2.

The signal 2, Fig.4, allows the viewing of the two-flow modes present in the bistable flow, by means of the instantaneous velocity values. In Fig. 4-a, the measured signals from probes 1 and 2 are presented. After 5 seconds a switch on the flow mode can be observed. This switch is better viewed after applying wavelet filter for a band of frequencies 0-3.9 Hz, Fig. 4-b.

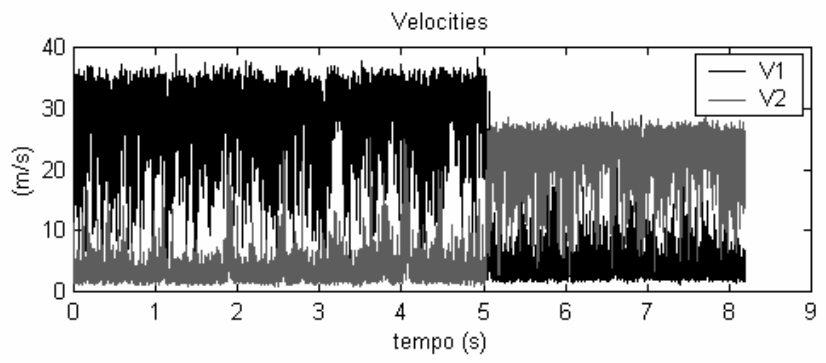

(a)

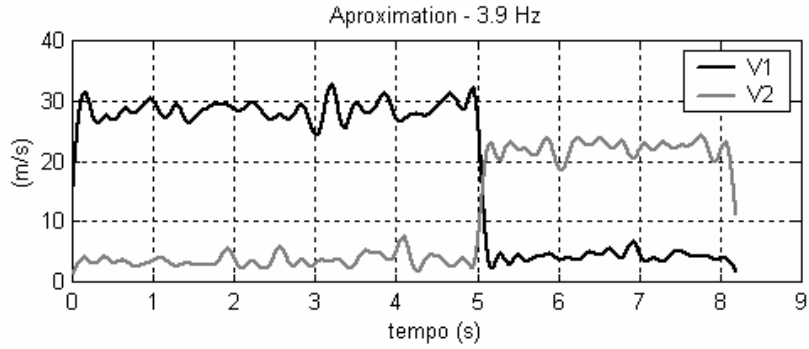

(b)

Figura 4. Characteristics of the signal 2 signal and corresponding discrete wavelet transforms. (a) measured signals; (b) Wavelet filtered signals (low pass filter, $3,9 \mathrm{~Hz}$ ).

Figure 5 shows the continuous wavelet spectrum, which represents the energy distribution of the velocities in time and frequency domain simultaneously. Figure 5-a shows that the higher peaks occur between 20 and $100 \mathrm{~Hz}$, and that they are associated with the higher velocities values during the first 5 seconds in velocity V1. For velocity V2, Fig. 5-b shows lower energy values for the first 3.5 seconds. This is associated with the lower velocity values. After this time the energy in the spectrogram increases, meanly, between 20 and $100 \mathrm{~Hz}$, and stay in this mode until the end of the record. For velocity V2, the energy level increases before of the change of the mode. This occurs because of the jump in the velocity V2, at about 4 seconds (see Fig.4).

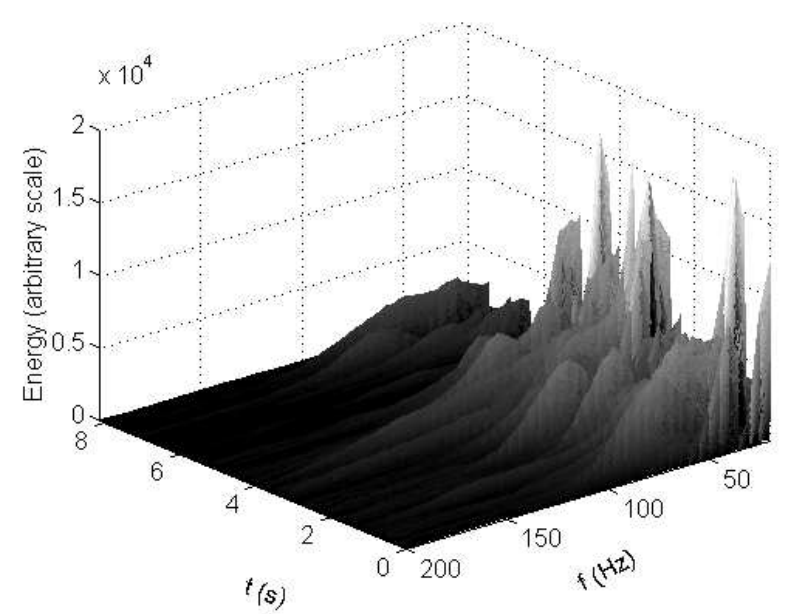

(a)

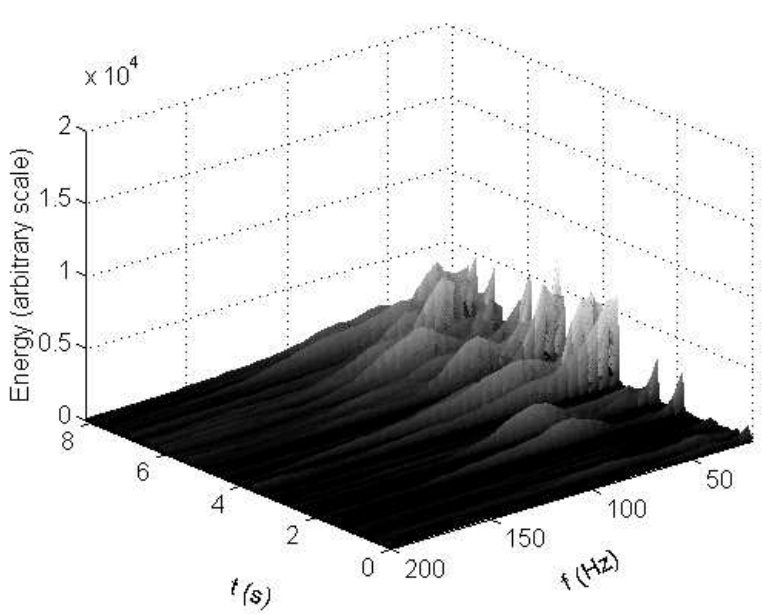

(b)

Figure 5. Velocity signal Spectrograms - signal 2: (a) V1, (b) V2.

For the study of the flow behavior, the signals V1 and V2 were separated in two clusters: the first one named here mode A and the second one, mode B. The first cluster is composed by the values measured from zero to $4.1 \mathrm{~s}$, corresponding to 32,768 elements. The second one has 16,384 elements, from 5.5 to $7.6 \mathrm{~s}$. Using this separation, the statistical functions were calculated for each one, as given in Table 2. It can be observed that the switching between the velocities obtained from the probe 1 and the probe 2 carries the statistical characteristics from each mode. This behavior is associated to the switching in the gap flow direction.

Table 2. Statistical characteristics of the signal 2.

\begin{tabular}{|l|c|c|c|c|}
\hline & \multicolumn{2}{|c|}{ mode A } & \multicolumn{2}{c|}{ mode B } \\
\hline & V1 & V2 & V1 & V2 \\
\hline Mean Velocity & 28.39 & 3.59 & 4.37 & 22.23 \\
\hline Standard deviation & 5.48 & 2.43 & 2.62 & 3.70 \\
\hline Skewness & -1.57 & 3.60 & 3.02 & -1.83 \\
\hline Kurtosis & 5.33 & 20.40 & 16.71 & 6.71 \\
\hline
\end{tabular}


The velocity spectra, shown at Fig.6, calculated for the two signals at each mode, allow identifying the presence of a mean frequency about $70 \mathrm{~Hz}$ for the mode $\mathrm{A}$, and about $60 \mathrm{~Hz}$ for the mode B. These frequencies correspond to Strouhal number of 0.16 and 0.14 , respectively, calculated with reference velocity. For mode $B$ it is possible to identify another frequency energy peak at $180 \mathrm{~Hz}$ for velocity V2, which corresponds to $\mathrm{St}=0.42$. A similar frequency would be expected for the mode A, at velocity V1. Probably it was not identified due to the probe location. Thereby, one can conclude that the velocity $\mathrm{V} 1$ in mode $\mathrm{A}$ and velocity $\mathrm{V} 2$ in mode $\mathrm{B}$ are related with the narrow wake whilst the velocity V2 in mode A and velocity V1 in mode B are related with the wide wake.

The values found do not agree with those presented by Alam et al., 2003. This can be produced by the high blockage ratio of the tunnel in the present experiments. Nevertheless, the two expected bistable mode shedding frequencies were found.

The signal 3 obtained with the probes positioned $70 \mathrm{~mm}$ downstream the tubes centerline does not show the bistable effect, characterizing, therefore, the single wake flow. This behavior is confirmed using the wavelet discrete transform, at Fig.7 b, and by continuous wavelet spectra (Fig.8), where the changing characteristic behavior is not observed for the both signals. Thus, one can consider that the two probes are measuring the velocity inside the same wake. The statistical analysis was made without dividing the signal in its two characteristic modes because it was only possible to measure velocities in the wide wake due to the biased gap flow, at this probe location.

From Tab. 3 one can verify that the measured values in both probes have characteristics related to those of the wide wakes. At this distance the narrow wake characteristic is already vanished. Figure 9 shows the energy spectrum, where the $70 \mathrm{~Hz}$ peak, related to the wide wake, is confirmed.

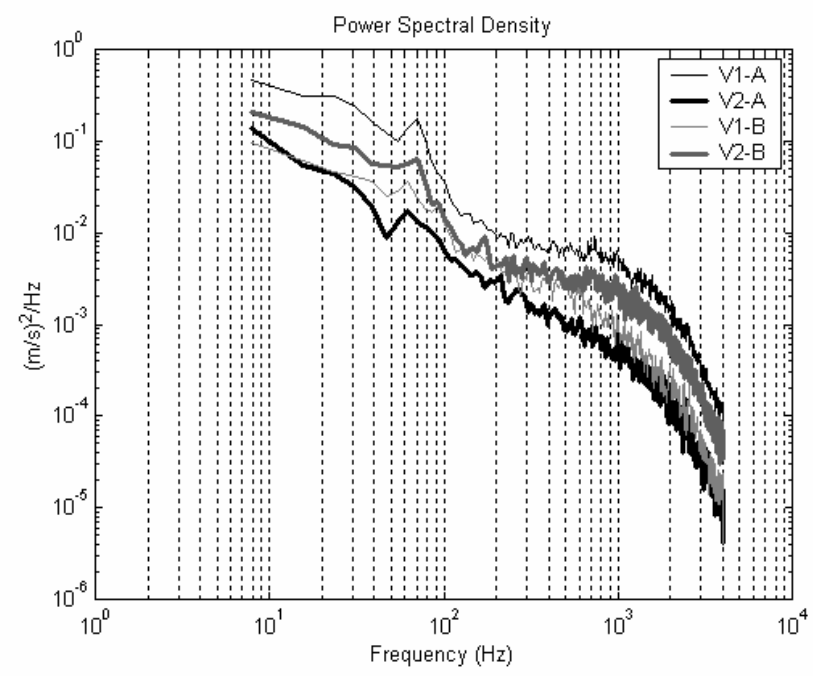

Figure 6. Power spectral densities of the velocity fluctuation.

Table 3. Statistical characteristics of the signal 3.

\begin{tabular}{|c|c|c|}
\hline & V1 & V2 \\
\hline Mean velocity & 7.65 & 6.10 \\
\hline Standard deviation & 3.84 & 3.52 \\
\hline Skewness & 1.40 & 1.81 \\
\hline Kurtosis & 6.23 & 7.32 \\
\hline
\end{tabular}
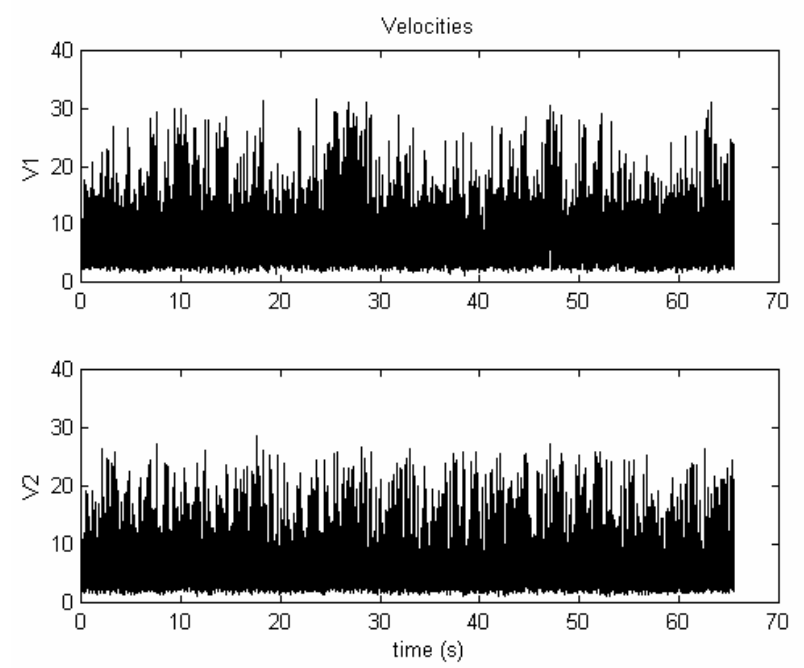

(a)
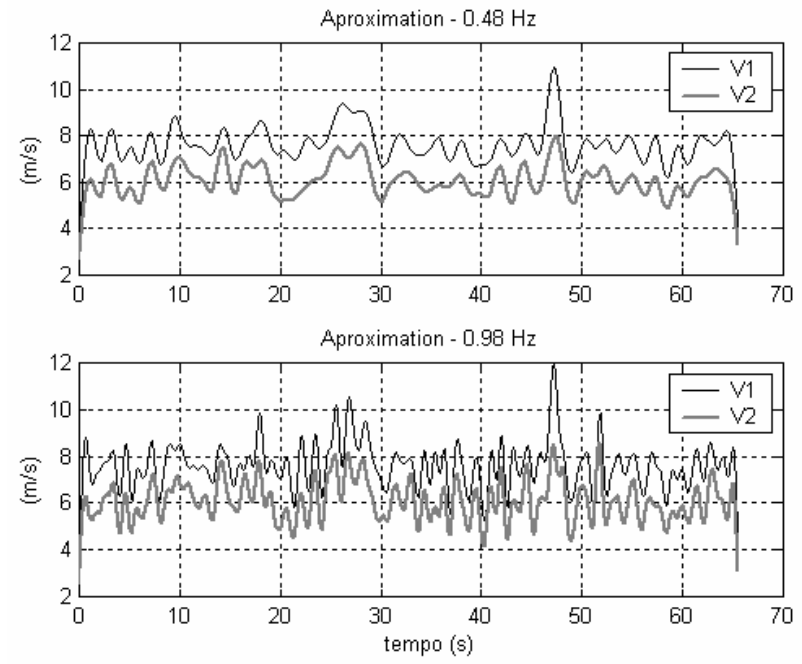

(b)

Figure 7. Characteristics of the signal 3 and corresponding discrete wavelet transforms. (a) Measured signals; (b) Wavelet filtered signals (low pass filter, $0.48 \mathrm{~Hz}$ and $0.98 \mathrm{~Hz}$ ).

\section{Tube Bank}

To study the bistable phenomenon inside tube banks, velocity time series behind the center tube of the third row were obtained, according to Fig. 3-b. The first one, acquired during 21s with an sampling frequency of $25 \mathrm{kHz}$ during a starting transient, is shown in Fig. 10-a. The respective wavelet filtered signals (low pass filter, $12.2 \mathrm{~Hz}$ ) were shown at Fig. 10-b. The switching between the two modes, characterized by the mean velocity variations, is impossible to be identified in Fig. 10-a, but is clearly visible in Fig. 10-b. Figure 11 shows the spectrograms for the first 5 seconds of the velocity signals, corresponding to the transient part. In these spectrograms the switches shown in Fig. 10-b are viewed as changes in the energy level. Between 2.5 and 3.5 seconds the velocity V1 has a high energy level (Fig. 10-b) whilst the velocity V2 keeps its original value, lower than $\mathrm{V} 1$. In the spectrogram, it can be seen that during this time interval, for $\mathrm{V} 1$ the energy has low values whilst for V2 there are several peaks, meanly, in the range from 10 to $100 \mathrm{~Hz}$. 


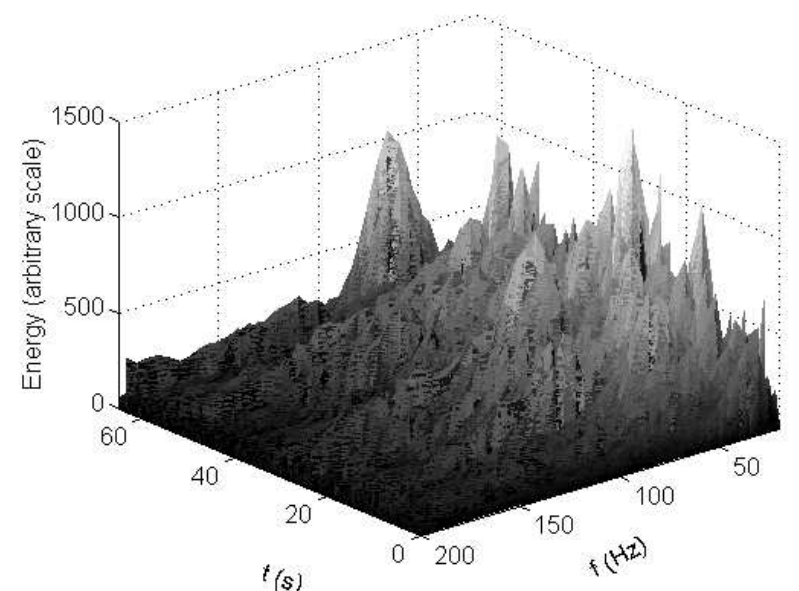

(a)

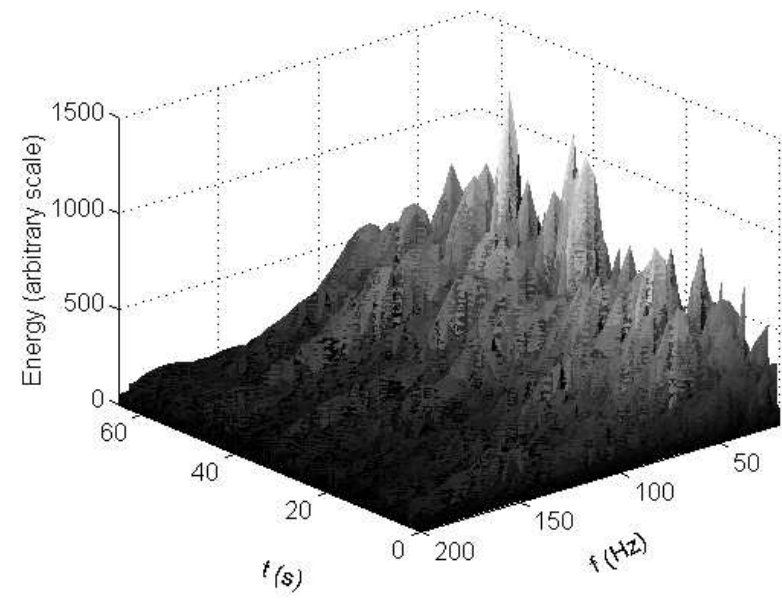

(b)

Figure 8. Spectrograms of the velocities - signal 3: (a) V1, (b) V2.

An acquisition where the bistable phenomenon is present was selected for the study of the mode characteristics (Fig. 12). In the wavelet discrete transform, it is possible to identify the moment when the mean velocity V2 changes. It is also possible to identify that this change is associated with a positive pressure jump at the same time location. This occurs for all cases, including two tubes side-by-side.

Figure 13 shows the spectrograms generated near the time location where the flow mode switch occurs. While for velocity V1 an almost uniform behavior is observed, since no significant variation on the velocity behavior is observed (Fig. 12). For the velocity V2, the spectrogram shows a variation on the energy, at $19 \mathrm{~s}$, where the velocity jump occurs

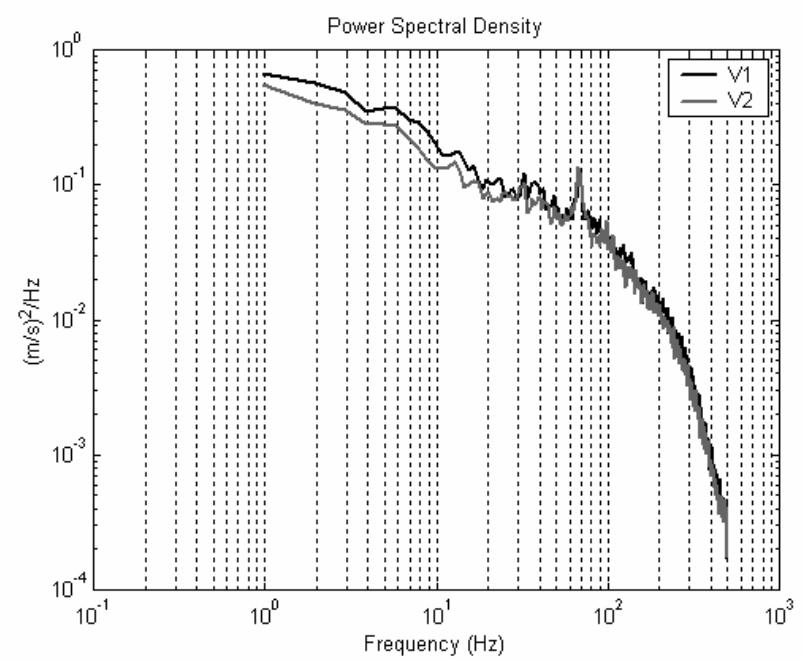

Figure 9. Fourier spectra of the signal 3.
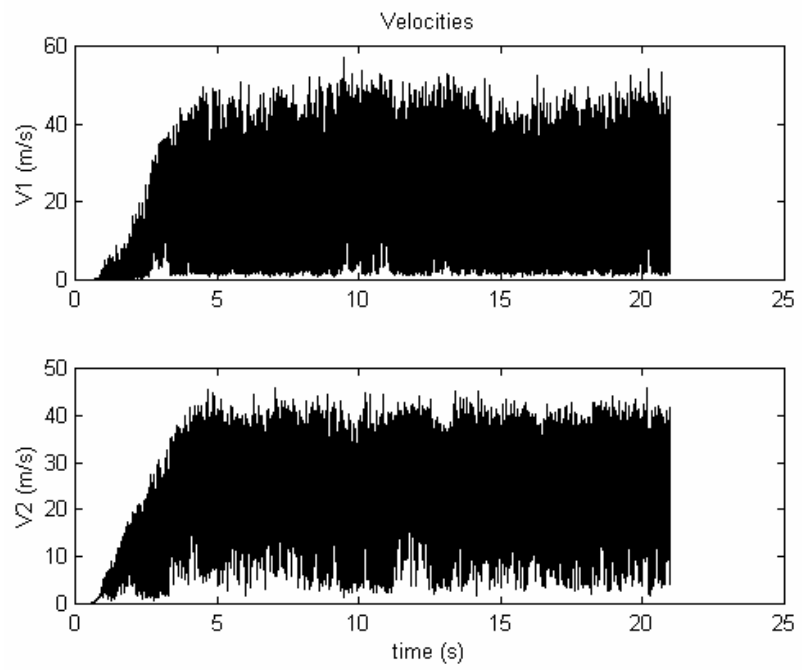

(a)

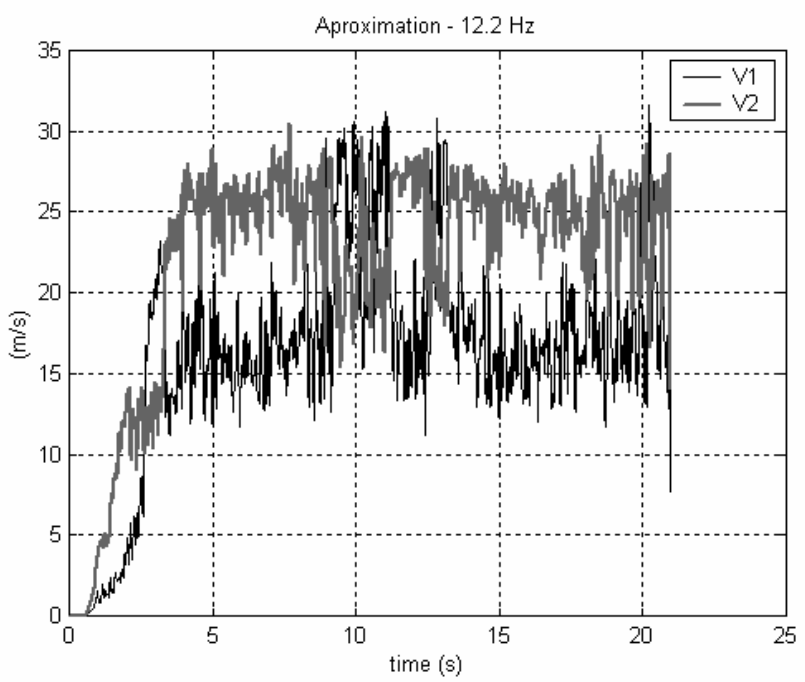

(b)

Figure 10. (a) Instantaneous velocities inside the tube bank and (b) corresponding wavelet discrete transform - first acquisition. 


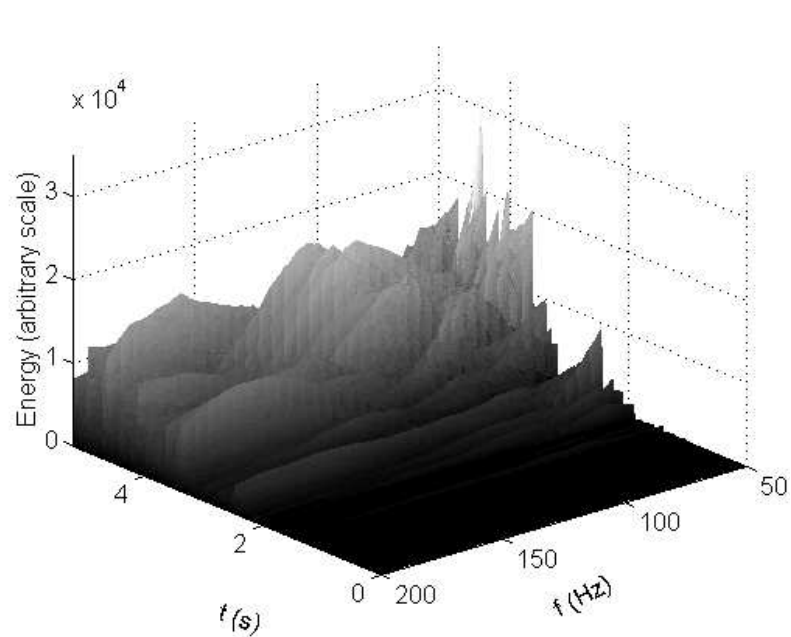

(a)

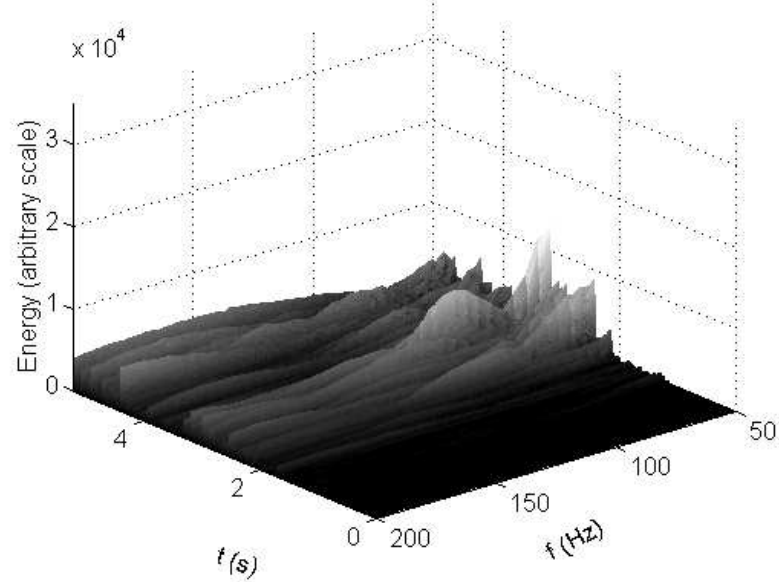

(b)

Figure 11. Spectrograms for the first 5 seconds of the velocity plots of Fig. 10: (a) V1, (b) V2.
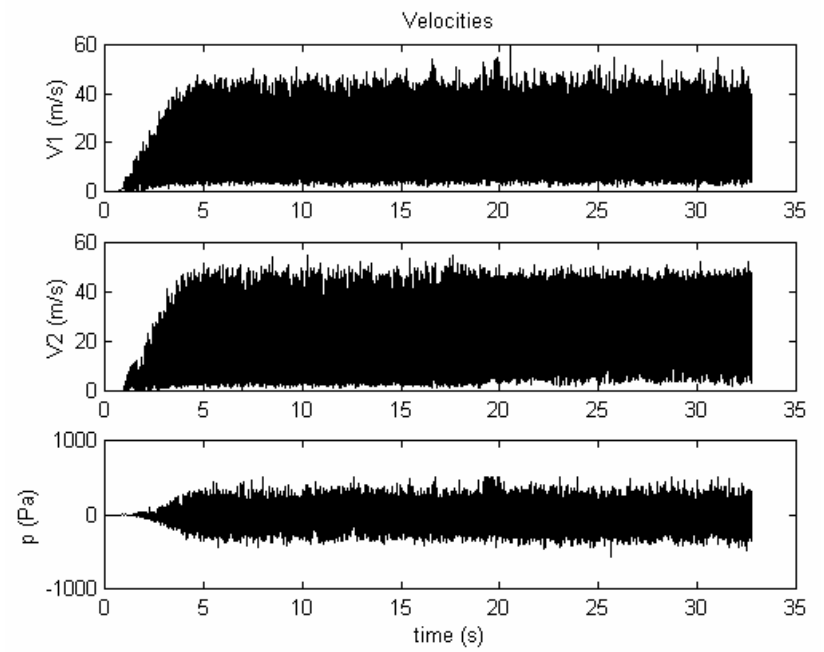

(a)

Figure 12. Tube bank, second acquisition: (a) instantaneous velocities and pressure, (b) corresponding wavelet discrete transform.
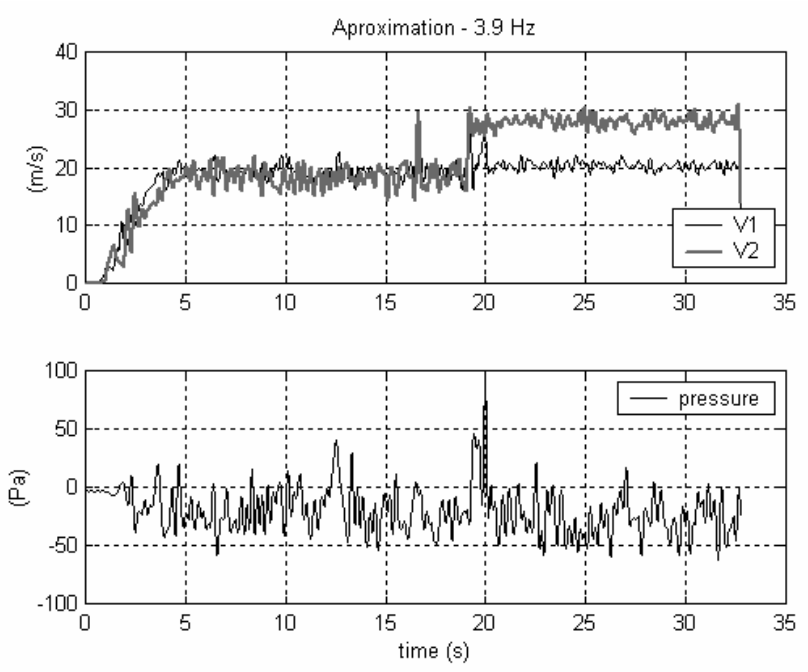

(b)

Figure 12. (Continued).

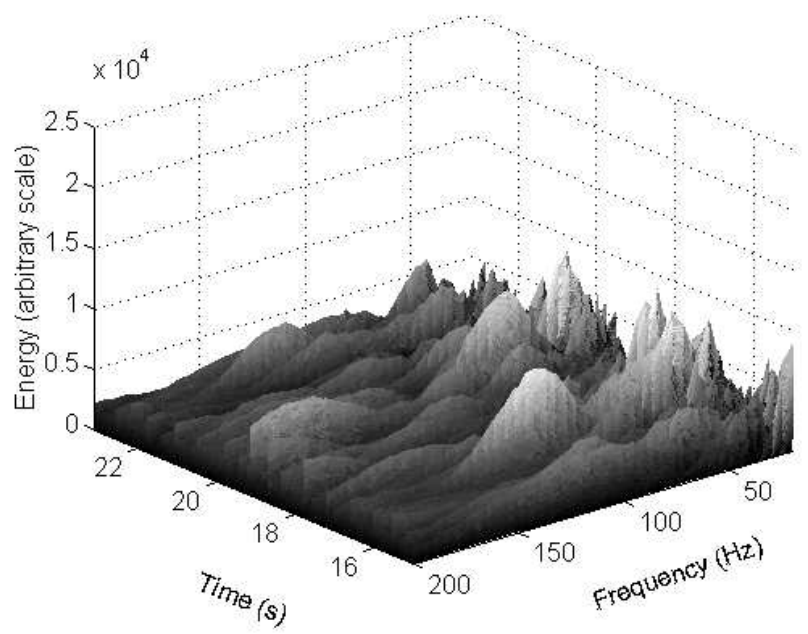

(a)

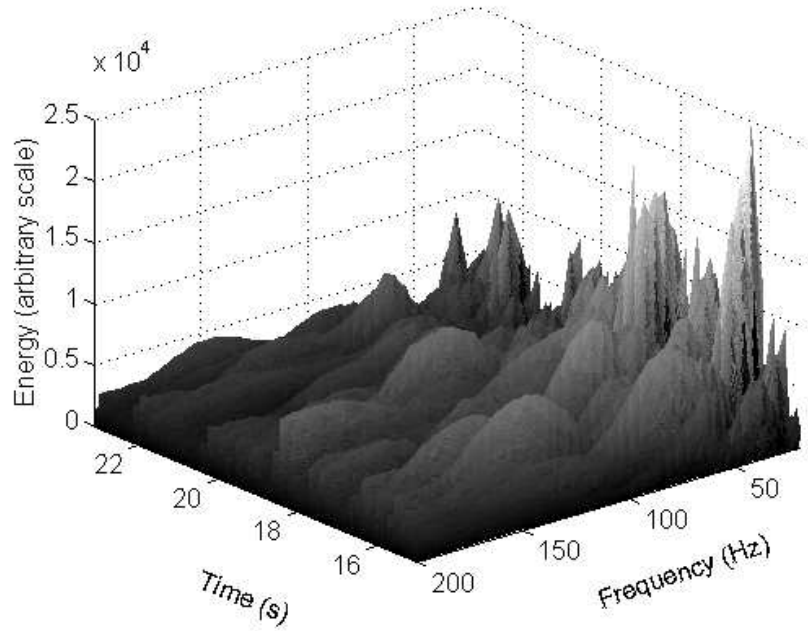

(b)

Figure 13. Tube bank, second acquisition - spectrograms of velocities: (a) V1; (b) V2. 
Table 3. Statistical characteristics of the second acquisition for tube bank - mode A and B.

\begin{tabular}{|l|c|c|c|c|c|c|}
\hline & \multicolumn{3}{|c|}{ mode A } & \multicolumn{3}{c|}{ mode B } \\
\hline & $\mathrm{V} 1$ & $\mathrm{~V} 2$ & $\mathrm{p}$ & $\mathrm{V} 1$ & $\mathrm{~V} 2$ & $\mathrm{p}$ \\
\hline Mean velocity & 19.22 & 18.28 & -16.46 & 20.36 & 28.02 & -29.02 \\
\hline Standard deviaton & 8.76 & 9.81 & 108.84 & 8.12 & 8.53 & 113.80 \\
\hline Skewness & 0.48 & 0.65 & 0.28 & 0.39 & -0.43 & 0.14 \\
\hline Kurtosis & 2.49 & 2.64 & 3.25 & 2.73 & 2.73 & 3.19 \\
\hline
\end{tabular}
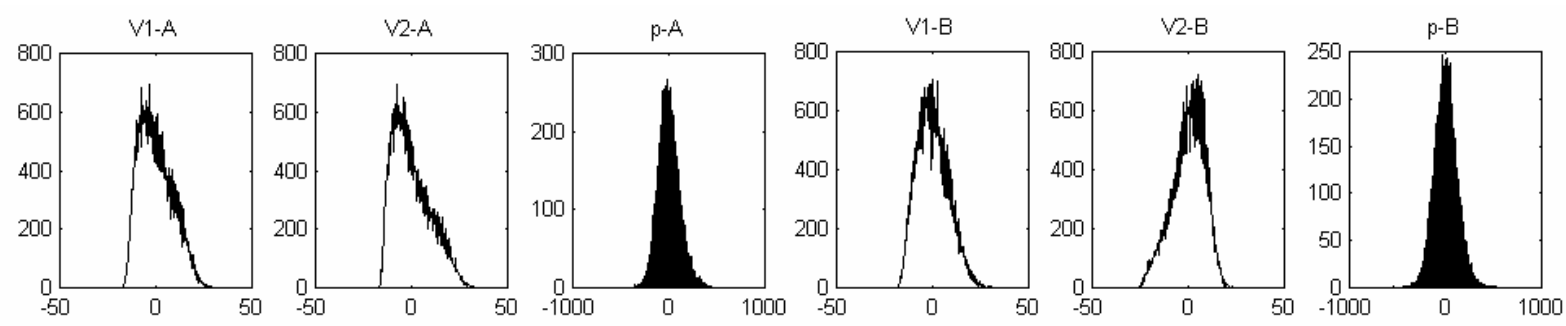

Figure 14. Probability density functions of the velocity and pressure signals for second acquisition.

In order to characterize the two-mode flow, the steady part of the signal was divided into two groups: the first one will be called here mode A, from $8 \mathrm{~s}$ to $16.2 \mathrm{~s}$, and the second one, called mode $\mathrm{B}$, from 22 to $30.2 \mathrm{~s}$. Their statistical characteristics are shown at Tab. 3 and Fig. 14 shows the probability density functions. The reference velocity measured upstream with the Pitot tube was $7.24 \mathrm{~m} / \mathrm{s}$.

In contrast to the side-by-side tube array, the statistical features do not change between the two modes. The most important change is the mean velocity jump at velocity V2. The mean velocity variation is associated to local pressure variation, thus drag coefficient variations are expected. This is responsible for the dynamic response on the tubes.

No important peaks can be observed in the velocity and pressure spectra shown in Fig. 15. This is the behavior expected inside in line tube banks, especially after the third row, according to the results by Ziada et al., 1989.

Additional data acquisitions were made inside the tube bank, changing the initial conditions and introducing flow disturbances. In some cases, new flow modes were generated, in other cases, the original flow mode did not change. In all the cases investigated, a rigorous evaluation was performed to identify the presence of instabilities.

\section{Discussion and Conclusions}

In this paper, the experimental study of the mean characteristics of the phenomenon named bistable flow is presented. This phenomenon has been found in the flow on two side-by-side cylinders, being characterized by the flopping of the wake to the back side of one of the cylinders, which moves to the back of the second cylinder in a random way, after assuming an stable condition. More recently, it was found evidences of its presence in aligned tube banks. This phenomenon can become an important source of dynamic instabilities, since it alternates the lift and drag coefficients, alternating the structure dynamic response.

These two kinds of arrays were experimented in a wind channel, using hot wires as experimental technique and analyzed by means of statistical, spectral and wavelet tools.

The features of the hot wire signals measured in several regions of the wake of two tubes confirm the results from the literature of the randomness of the flopping process. The origin of this phenomenon, however, is not completely understood. It has its origins in the velocity, and thus in the vorticity, fluctuations of the neighboring wakes. It is expected that, in the beginning of the process, the wakes are generated independently from each other. If the neighboring vortices have the same phase, the fluctuation will make the wake grow. If the P/D-ratio is greater than one, the resulting wake will not behave as on a single bluff body due to the flow through the gap between the tubes, rather it will remain in the back side of one tube until a new disturbance destabilizes the wake and the process starts again.

In tube banks, the flopping is influenced by the highly disordered flow after the second and third rows, which in some geometries do not allow the observation of a characteristic frequency. However, the vortex shedding process still occurs. If the wake interaction, as described for two tubes placed side by side, occurs, the flopping will be strongly influenced by the presence of the tubes of the next row, so that there is no space for the flopping formation as in the wake of two or more tubes. The consequence is that the flopped flow will be directed up or downwards, that means to a direction parallel to the tubes axes, giving a strong three dimensional characteristic to the flow through tube banks.

An additional question may arise when the tube bank flow is studied with a view to the heat exchangers analysis. This being about the possibility of the flopping becomes harmful, and therefore must be avoided or, at least controlled or if its effects can be profitable by, for instance, enhancing the heat transfer process.

Nevertheless, by observing the experimental results, it becomes evident that special care should be taken for the Strouhal number range where these instabilities can occur. Therefore, new studies, including flow visualizations, are necessary to enhance the understanding of the bistable phenomenon inside tube banks, mainly on the first rows.

The observation and identification of the instabilities by using hot wire technique was only possible by means of the application of the wavelet transforms, since the Fourier spectral analysis is not appropriate for identifying phenomena where intermittent processes are present. Therefore, wavelet transforms, either continuous or discrete, are very suitable tools for the analysis of the studied problem. 


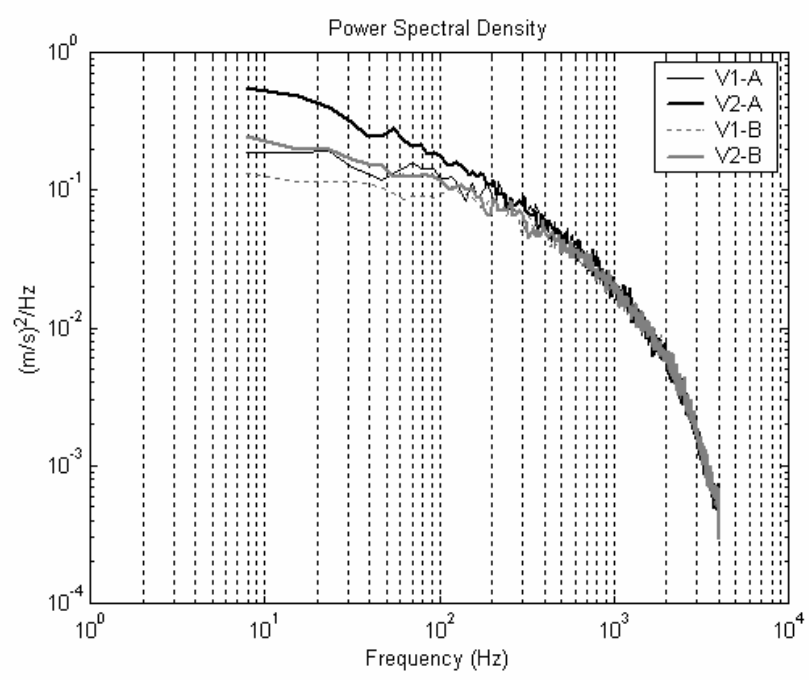

(a)

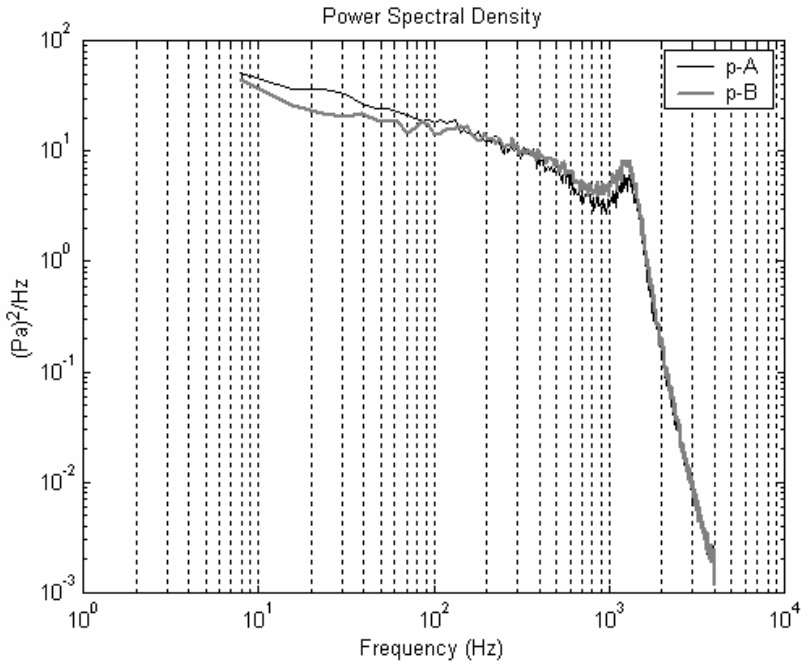

(b)

Figura 15. Fourier spectra of velocity and pressure signals, modes A and B.

\section{Acknowledgements}

Authors gratefully acknowledge the support by the CNPq Brazilian Scientific and Technological Council, under the grants 414216/90-3, 400180/92-8 and 520986/1997-0.

Cláudio R. Olinto thanks CAPES, Ministry of Education, Brazil, for granting him a fellowship (PICDT).

\section{References}

Alam, M. M., Moriya, M. and Sakamoto, H., 2003, "Aerodynamic characteristics of two side-by-side circular cylinders and application of wavelet analysis on the switching phenomenon", Journal of Fluids and Structures, Vol. 18, pp. 325-346.

Goulart, J. N., 2004, "Experimental study of the pressure and velocity fields in tube banks with baffle plates" (in Portuguese) M. Eng. Dissertation, PROMEC - Federal University of Rio Grande do Sul, Porto Alegre, Brazil.

Guillaume, D. W. and LaRue, J. C., 1999, Investigation of the flopping regime with two-, three- and four-cylinder arrays, Experiments in Fluids, Vol. 27, pp. 145-156.

Indrusiak, M. L. S. and Möller, S. V., 2004 "Wavelet analysis of experimental turbulence time series", IV Escola de Primavera de Transição e Turbulência, ABCM, Porto Alegre.

Indrusiak, M. L. S., Goulart, J. N. V., Olinto, C. R. and Möller, S. V., 2003, "Wavelet time-frequency analysis of accelerating and decelerating flows in a tube bank", Transactions of the $17^{\text {th }}$ International Conference on Structural Mechanics in Reactor Technology (SMiRT 17), Prague, Czech Republic.

Kim, H. J. and Durbin, P. A., 1988, Investigation of the flow between a pair of circular cylinders in the flopping regime, Journal of Fluid Mechanics, Vol. 196, pp. 431-448.

Le Gal, P., Peschard, I., Chauve, M. P. and Takeda, Y., 1996, Collective behavior of wakes downstream a row of cylinders, Physics of Fluids, Vol. 8, pp. 2097-2106.

Sumner, D., Wong, S. S. T., Price, S. J. and Païdoussis, 1999, Fluid Behaviour of side-by-side circular cylinders in steady cross-flow, Journal of Fluids and Structures, Vol. 13, pp. 309-338.

Williamson, C. H. K., 1985, Evolution of a single wake behind a pair of bluff bodies, Journal of Fluid Mechanics. Vol. 159, pp. 1-18.

Zdravkovich, M. M. and Stonebanks, K. L., 1988, Intrinsically nonuniform and metastable flow in and behind tube arrays, International Symposium on Flow-Induced Vibration and Noise, Chicago, Vol 1, pp. 6173.

Zdravkovich, M. M., 1977, Review of flow interference between two circular cylinders in various arrangements, Journal of Fluids Engineering (Transactions of the ASME), December, pp. 618-633

Zhou, Y., Zhang, H. J. and Yiu, M. W., 2002, The turbulent wake of two side-by-side circular cylinders, Journal of Fluid Mechanics, Vol. 458, pp. 302-332.

Ziada, S., Oengören, A and Bühlmann, E. T., 1989, "On acoustical resonance in tube arrays. Part I: Experiments", Journal of Fluids and Structures, Vol. 3, pp.293-314. 